\title{
Exercício físico em grupo para indivíduos com Doença de Parkinson: Revisão integrativa da literatura
}

\author{
Group physical exercise for individuals with Parkinson's disease: \\ Integrative literature review
}

\author{
Lílian de Fátima Dornelas ${ }^{1}$
}

${ }^{1}$ Fisioterapeuta, Doutora em Ciências da Reabilitação

Centro Especializado em Reabilitação - CER III, Prefeitura Municipal de Uberlândia/MG

\begin{abstract}
Resumo
Introdução: O exercício físico em grupo para indivíduos com a Doença de Parkinson pode melhorar a condição física, a autoestima e otimizar os atendimentos gerando economia de recursos. Objetivo: Analisar os benefícios do exercício físico nos sintomas motores e não motores de indivíduos com Doença de Parkinson. Metodologia: Foi realizada uma busca bibliográfica nas bases Medline, Pubmed, Scopus, Web of Science e One File (Gale), utilizando os termos, exercício físico em grupo (group physical exercise) combinada com Doença de Parkinson (Parkinson's Disease), função motora (motor function) e função não motora (no motor function), elegendo artigos escritos em português e inglês que tratavam de exercícios físicos em grupo, em ambiente terrestre, sob desfechos motores e não motores, limitados nos anos de 2012 a 2016. Os trabalhos incluídos no estudo foram analisados segundo a qualidade metodológica pela escala PEDro. Resultados: Sete artigos foram selecionados para integrar a revisão após serem submetidos à criteriosa avaliação por dois avaliadores cegados (Kappa=0,86). As intervenções em grupo que utilizaram dança, Tai chi, Yoga, pistas sensoriais, circuitos aeróbicos e com resistência física apresentaram melhores respostas quanto aos sinais motores e não motores. Conclusão: O exercício físico em grupo é uma estratégia que associa benefícios motores e não motores, por associar a vivência de indivíduos que são acometidos pela mesma enfermidade, com exercícios sob abordagens que estimulam estratégias cognitivas, sensoriais e motoras.
\end{abstract}

Palavras chave: Doença de Parkinson; Fisioterapia; Exercício físico. 


\section{Abstract}

Introduction: Group physical exercise for individuals with Parkinson's disease can improve physical condition, self-esteem and optimize care giving resources savings. Objective: To analyze the benefits of physical exercise in the motor and non-motor symptoms of individuals with Parkinson's Disease Methods: A bibliographic search was performed on the Medline, Pubmed, Scopus, Web of Science and One File (Gale) databases using the terms group physical exercise combined with Parkinson's Disease, motor function (motor function) and non-motor function (motor function), choosing articles written in Portuguese and English that dealt with group physical exercises in terrestrial environment, under motor and non-motor outcomes, limited in the years 2012 to 2017. Included in the study were analyzed according to methodological quality by the PEDro scale. Results: Seven articles were selected to integrate the review after being submitted to the careful evaluation by two blinded evaluators (Kappa $=$ 0.86). Group interventions that used dance, Tai Chi, Yoga, sensory cues, aerobic circuits and physical resistance presented better responses regarding motor and non-motor signals. Conclusion: The group approach is a strategy that associates motor and non-motor benefits by associating the experience of individuals who are affected by the same disease, with exercises under approaches that stimulate cognitive, sensorial and motor strategies.

Keywords: Parkinson Disease; Physiotherapy; Physical exercise

\section{Introdução}

A Doença de Parkinson (DP) resulta no comprometimento das vias neurodopaminérgicas da região dos gânglios da base, devido à deficiência de dopamina levando a um complexo quadro clínico․ É uma das doenças neurodegenerativas mais comuns e atinge todos os grupos étnicos e classes socioeconômicas, estimando uma prevalência de 100 a 200 casos por 100.000 habitantes.

Os sintomas da DP resultam da atividade reduzida das células da região compacta da substância negra que podem ser desde acometimentos motores, como a rigidez, bradicinesia, tremor e alteração postural e também sintomas não motores, tais como sintomas de depressão, apatia, problemas cognitivos, distúrbios do sono, que podem impactar negativamente na vida diária e na qualidade de vida dos indivíduos acometidos por esta patologia ${ }^{2}$. Esta possibilidade de acometimentos pode estar relacionada com os caminhos que a dopamina percorre no cérebro, que abrange a via nigroestriatal e o movimento mediador que são os mais visivelmente afetados na DP e a via não-estriatal que tem explicado os sinais não motores associada à $\mathrm{DP}^{1,2}$.

Nesse sentido, o olhar biopsicossocial é fundamental para a avaliação e a instituição de condutas em pacientes com DP. A Classificação Internacional de Funcionalidade, Incapacidade e Saúde (CIF) permite abordar os diferentes constructos relacionados à funcionalidade e à incapacidade do indivíduo, considerando suas deficiências em estrutura e função, bem como limitações em atividade e participação. Os fatores ambientais e pessoais impactam diretamente sobre os demais domínios, possuindo igual importância na funcionalidade do indivíduo ${ }^{3}$.
Nas últimas décadas, o olhar da fisioterapia tem se baseado para não somente para a estrutura e função do corpo, mas também para a funcionalidade do indivíduo, e o principal objetivo em indivíduos com DP é maximizar a capacidade funcional e minimizar complicações secundárias ${ }^{4}$. Keus e colaboradores ${ }^{5}$ traçaram as principais recomendações para a reabilitação fisioterápica na DP: estratégias com estímulos para o treinamento da marcha, estratégias cognitivas para melhorar as transferências, exercícios para o equilíbrio, treinamento da mobilidade articular e força muscular para implementar a capacidade física.

Embora não se tenha evidências mostrando qual a abordagem é mais eficiente para pessoas com a DP, programas de atividade física têm sido documentados como uma influência positiva na DP, relacionado com a melhora de sintomas motores e não motores ${ }^{1,2,3,4}$. Estudos ${ }^{6,7,8}$ que, apoiam a prática do exercício físico apontam benefícios cardiovasculares, na função física, na saúde global e no bem-estar de indivíduos com DP. Além disso, o exercício físico tem sido recomendado para pacientes com doença de Parkinson independentemente da idade e do estágio da doença9 ${ }^{9}$.

Dentre os programas terapêuticos, os realizados em grupo podem trazer motivação e engajamento, aumentando a flexibilidade, força e resistência e tende a diminuir a depressão, tornando o paciente mais ativo no tratamento ${ }^{10}$. Além disso, o exercício físico realizado em grupo tem-se demonstrado eficaz apresentando algumas vantagens, tais como, promoção de um ambiente que estimula o convívio entre pessoas que tiveram suas vidas alteradas pela mesma enfermidade e que, portanto, apresentam limitações semelhantes, estímulo de outros pacientes à realização de atividades físicas benéficas e possibilidade de atendimento simultâneo de vários pacientes, gerando economia de recursos ${ }^{11}$. 
Pensando nisto, o presente estudo tem como pergunta norteadora: Quais são as práticas de exercício físico em grupo realizadas com indivíduos com DP e suas evidências? Assim, o objetivo é analisar os benefícios do exercício físico nos sintomas motores e não motores de indivíduos com DP.

\section{Metodologia}

O estudo caracteriza-se por uma revisão integrativa da literatura. Foram seguidas as seis etapas que compreende a revisão integrativa da literatura ${ }^{12}$ :

1. Identificação da questão de pesquisa para a elaboração da revisão integrativa;

2. Estabelecimento de critérios para inclusão e exclusão de estudos e busca na literatura;

3. Definição das informações a serem extraídas dos estudos selecionados;

4. Avaliação dos estudos incluídos na revisão integrativa;

5. Interpretação dos resultados;

6. Apresentação da revisão e síntese do conhecimento.

\section{Estratégia de busca}

Foi realizada uma busca bibliográfica inicialmente pelo Portal da Capes para identificar as bases de dados que mais citavam o tema, utilizando as palavras chave: exercício físico em grupo (group physical exercise) combinada com Doença de Parkinson (Parkinson's Disease), função motora (motor function) e função não motora (no motor function). Identificadas as bases eletrônicas mais frequentes: Medline, Pubmed, Scopus, Web of Science e One File (Gale) foram delimitados os critérios para a busca dos artigos, limitados nos anos de 2012 a 2016, artigos escritos em português e inglês e estudos experimentais.

\section{Seleção dos estudos}

Para a seleção dos artigos foram aplicados os seguintes critérios de inclusão e exclusão analisados independentemente por dois avaliadores, que os classificaram como "incluído", "excluído" ou "duvidoso". Nos casos em que houve divergência, os avaliadores discutiram até a obtenção de um consenso quanto à classificação.

Critérios de inclusão: população (indivíduos com Doença de Parkinson), intervenção (exercícios físicos realizados em conjunto, no ambiente terrestre), desfecho (função motora e não motora) e ensaios clínicos.

Critérios de exclusão: artigos que não realizaram atividade em grupo (o que inclui pelo menos mais de 2 pessoas), ausência de grupo controle, grupo controle sem a doença de Parkinson, artigos com animais, revisão de literatura, estudo de caso ou série de casos, tratamentos prioritariamente com abordagem medicamentosa ou com eletroterapia ou em ambiente aquático.

\section{Definições das informações extraídas}

As informações extraídas nos estudos selecionados foram definidas nos seguintes tópicos: autor/ano, objetivo, participantes, instrumentação, tipo de intervenção, desfechos, conclusão e serão apresentadas em um quadro na parte de resultados. Os estudos incluídos foram avaliados quando a qualidade metodológica de forma independente por dois avaliadores. Nos casos em que ocorreu divergência, os itens discrepantes foram revistos e discutidos até a obtenção de consenso a respeito da pontuação. Somente foram incluídos os estudos com alta qualidade metodológica (escore PEDro maior ou igual a 5).

\section{Avaliação dos estudos}

Existem diferentes escalas que auxiliam na avaliação dos estudos, tais como lista de Delphi, PEDro, OTSeeker, critérios de Maastricht, escala de Jadad, entre outras. A mais usada na área da reabilitação é a escala PEDro (PEDro scale, http://www.pedro.fhs.usyd.edu.au). Essa escala foi desenvolvida pela Physiotherapy Evidence Database para ser empregada em estudos experimentais e tem uma pontuação total de até 10 pontos, incluindo critérios de avaliação de validade interna e apresentação da análise estatística empregada. Para cada critério definido na escala, um ponto (1) é atribuído à presença de indicadores da qualidade da evidência apresentada, e zero ponto (0) é atribuído à ausência desses indicadores ${ }^{13,14,15,16}$.

A escala PEDro é composta pelos seguintes critérios: 1) especificação dos critérios de inclusão (item não pontuado); 2) alocação aleatória; 3) sigilo na alocação; 4) similaridade dos grupos na fase inicial ou basal; 5) mascaramento dos sujeitos; 6) mascaramento do terapeuta; 7) mascaramento do avaliador; 8) medida de pelo menos um desfecho primário em $85 \%$ dos sujeitos alocados; 9) análise da intenção de tratar; 10) comparação entre grupos de pelo menos um desfecho primário e 11) relato de medidas de variabilidade e estimativa dos parâmetros de pelo menos uma variável primária ${ }^{17}$.

\section{Resultados}

Como ilustrado na Figura 1, sete artigos foram incluídos na revisão integrativa. Todos obtiveram escore pela escala PEDro acima de 5, que foram avaliados por dois avaliadores cegados (Kappa>0,86), quanto a qualidade metodológica. 
Figura 1: Etapas seguidas durante a revisão integrativa da literatura.

\section{Busca pelas bases eletrônicas no Portal da Capes 1095 estudos}

Medline, Pubmed, Scopus, Web of Sicence, One Fiel (Gale): 185 artigos encontrados anos: 2012 a 2016

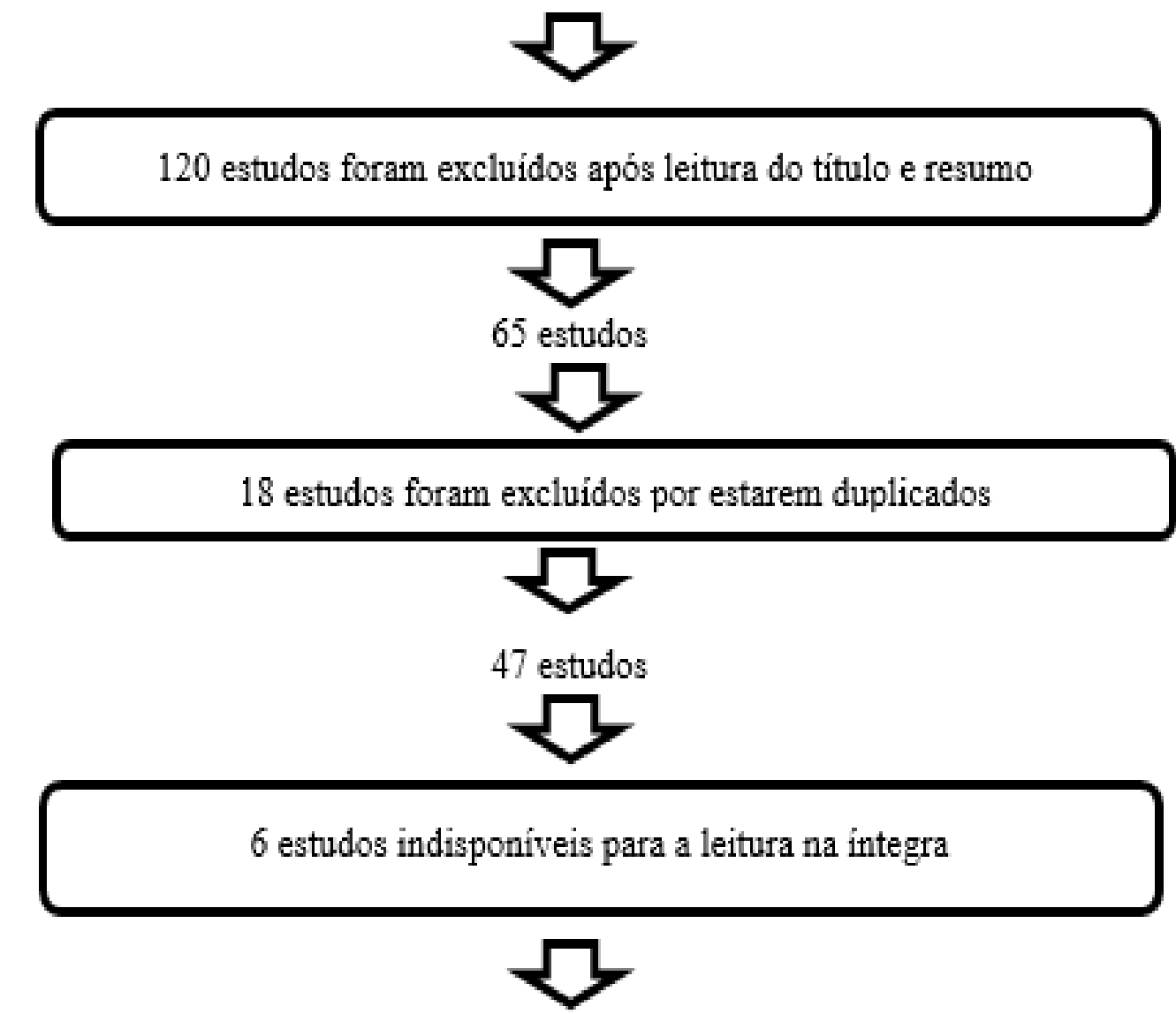

41 estudos foram avaliados quanto a qualidade metodológica

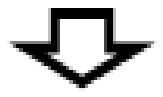

\section{7 estudos alcançaram escore PEDro igual ou acima de 5}

As informações apresentadas nos sete artigos selecionados nesta revisão integrativa da literatura estão apresentadas no quadro 1, quanto aos tópicos: autor/ano, objetivo, participantes, instrumentação, tipo de intervenção, desfechos, conclusão e qualidade metodológica. 


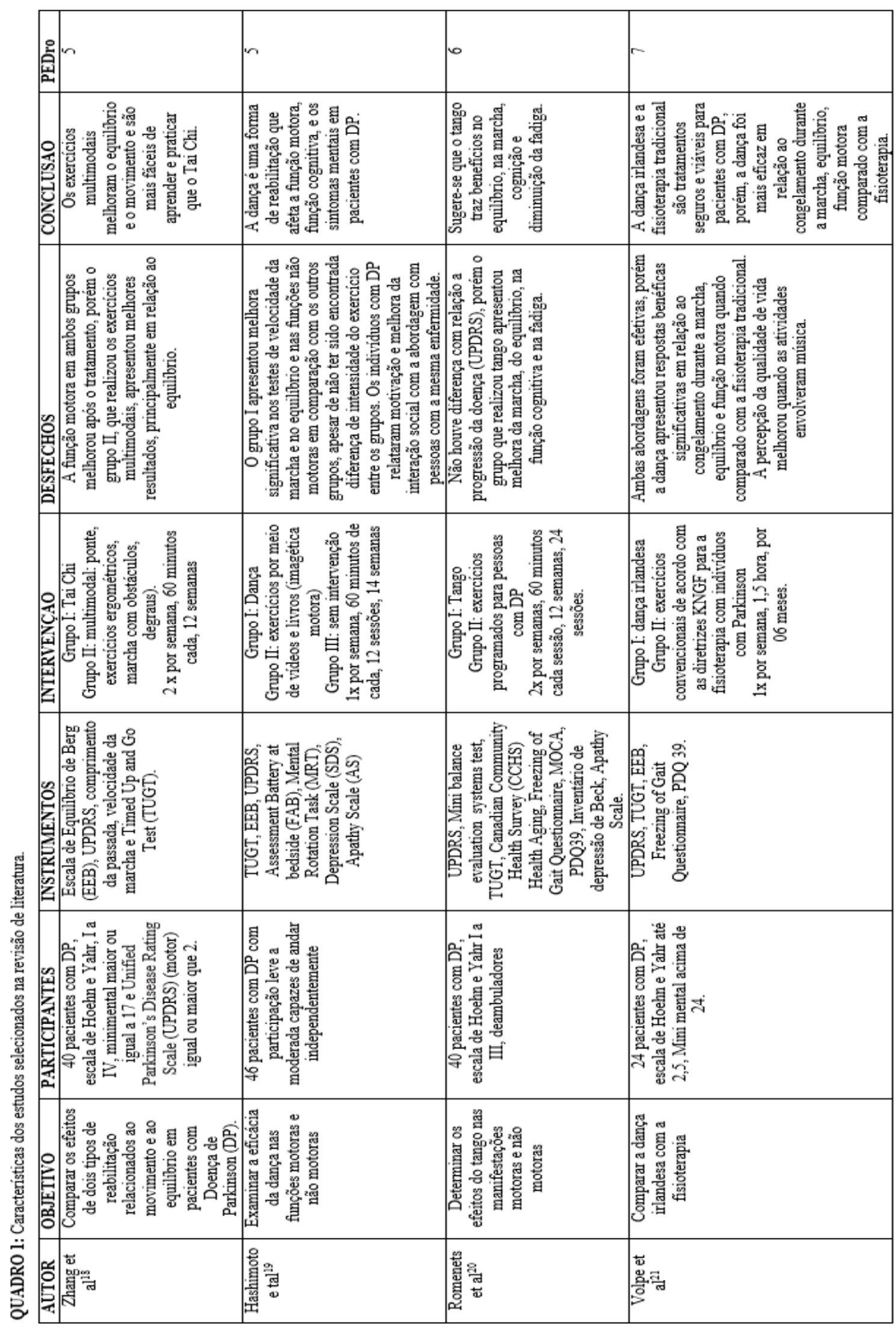




\begin{tabular}{|c|c|c|}
\hline P & $\mathrm{r}$ & $n$ \\
\hline 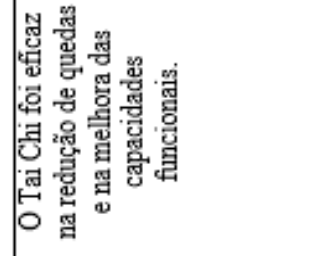 & 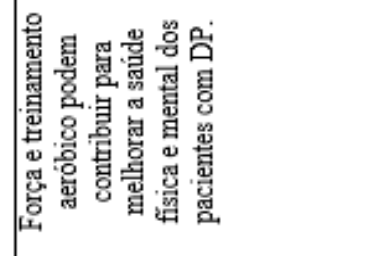 & 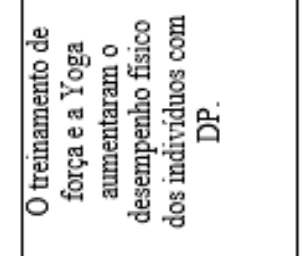 \\
\hline 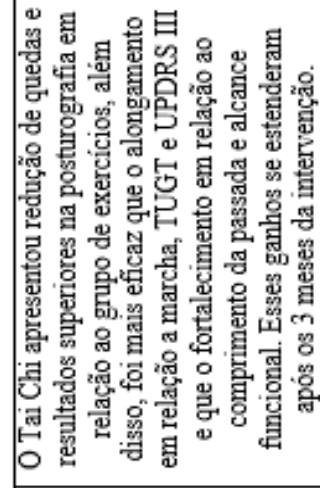 & 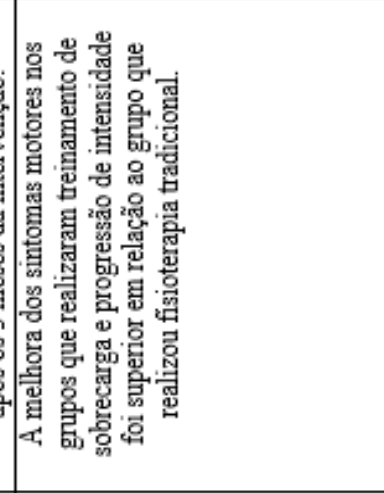 & 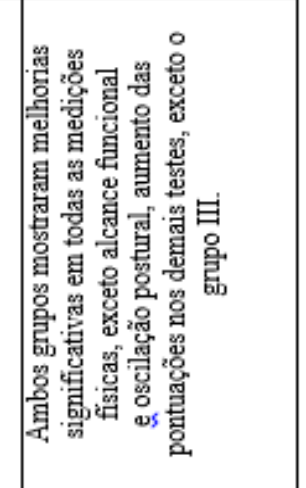 \\
\hline 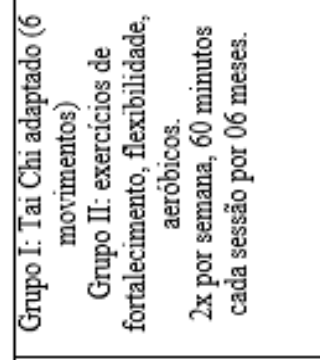 & 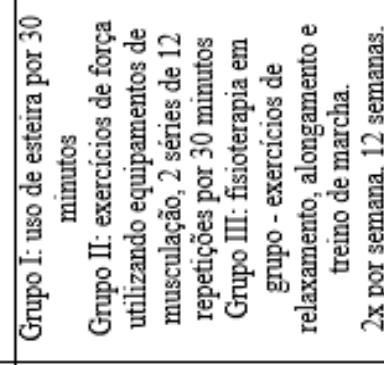 & 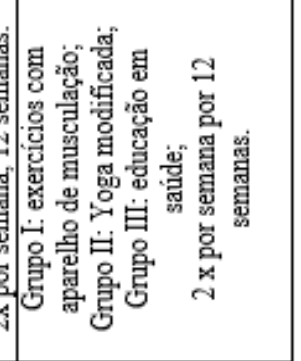 \\
\hline 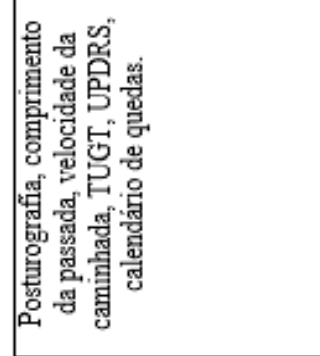 & 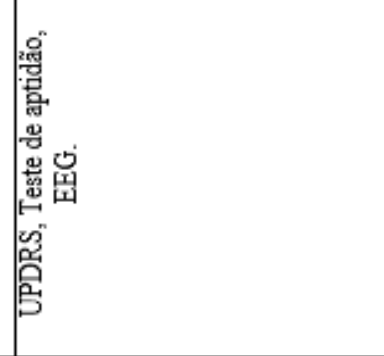 & 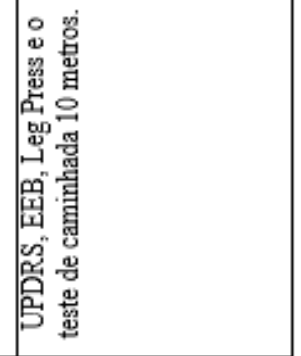 \\
\hline 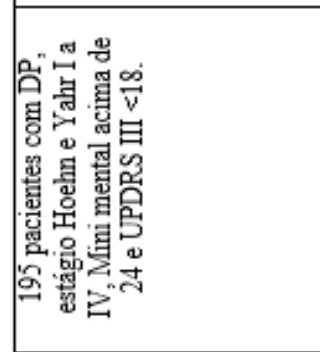 & 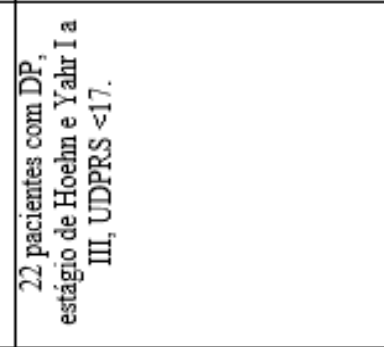 & 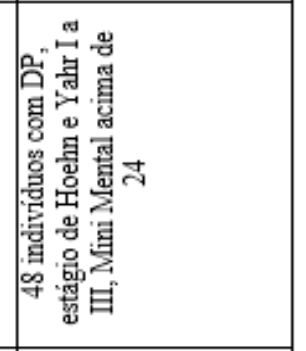 \\
\hline 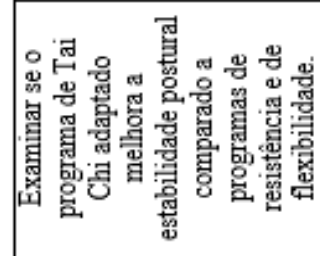 & 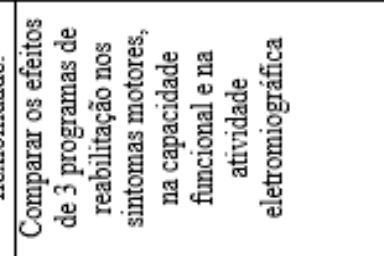 & 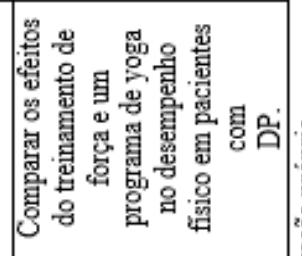 \\
\hline 䓌 & 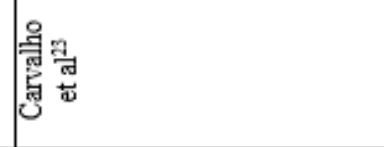 & 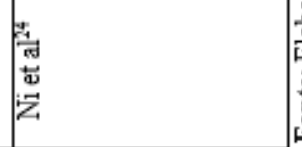 \\
\hline
\end{tabular}


Em relação à população pesquisada, a presença de uma amostra homogênea, ou seja, igual entre grupos no pré-tratamento, foi encontrada em todos os estudos ${ }^{18,19,20,21,22,23,24}$ e como critérios de inclusão para participação das atividades em grupo foram considerados o estágio da doença, a função cognitiva e o uso de medicação. Quanto ao estágio da DP, a escala Hoehn \& Yahr ${ }^{25}$ foi a utilizada como referência e os estágios de leve a moderada, os mais preponderantes. Já a função cognitiva, o Mini Mental ${ }^{26}$ (MEEM) e o Montreal Cognitive Assessment (MOCA) foram os questionários preferencialmente aplicados, considerando os valores superiores acima de $17^{18} \mathrm{e}$ $24^{21,22,24}$. O uso de medicamentos foi também um prérequisito para a inclusão do participante, e considerado a fase on durante a terapêutica, preferencialmente.

Os instrumentos de avaliação clínica mais usados para avaliação antes e após o exercício físico foram a Escala Unificada de Avaliação da Doença de Parkinson (Unified Parkinson's Disease Rating Scale - UPDRS) $)^{25,26}$ que, é considerado como padrão ouro na avaliação da progressão da doença de Parkinson, a Escala de Equilíbrio de Berg (EEB) ${ }^{26}$ para avaliar o equilíbrio, o Timed Up and Go Test (TUGT) ${ }^{26}$ que avalia as alterações posturais de tronco e membros inferiores, o teste de caminhada de dez metros ${ }^{26}$ para avaliar a velocidade da marcha e o Questionário de Qualidade de vida para Doença de Parkinson (PDQ$39)^{27}$ para verificar a percepção da qualidade de vida.

Os tipos de intervenção em grupo encontrados nos estudos foram principalmente a dança ${ }^{19,20,21}$, Tai chi $^{18,22}$, Yoga ${ }^{23}$, exercícios circuitados mesclando atividades aeróbicas e com resistência física, treino de equilíbrio e de marcha, com o uso de pistas sensoriais $^{23,24}$. Em geral, as intervenções em grupo apresentaram duração que variou entre seis e 24 semanas, intensidade de no mínimo 60 minutos e frequência de duas a três vezes por semana.

As medidas de desfecho frequentemente utilizadas nos estudos foram relacionadas à estabilidade postural, equilíbrio, "freezing", marcha, progressão da doença e qualidade de vida.

\section{Discussão}

O exercício físico parece trazer efeitos positivos para indivíduos com DP com relação aos sinais motores e não motores, destacando-se aqueles que envolvem atividades em grupo, com exercícios que trabalham com ritmo, movimentos amplos e repetitivos. Zhang et $\mathrm{al}^{18}$ apontam que, o exercício físico pode aumentar a neuroplasticidade e pode ser um fator neuroprotetor para indivíduos com DP. Dessa forma, o estímulo à atividade física se torna indispensável desde a fase inicial da doença, pois, proporciona melhora da funcionalidade e qualidade de vida, promovendo a saúde.

No entanto, ainda não está claro na literatura qual o tipo de exercício é mais eficaz para os indivíduos com DP, porém, observou-se nesta revisão integrativa que, ainda se enfatiza a busca pela melhora dos sinais motores. Zhang et al ${ }^{18}$ utilizaram o Tai Chi e o treino multimodal para membros inferiores, em indivíduos com DP e observaram que as atividades multimodais como, treinamento muscular para o CORE, atividades com obstáculos e com ciclo ergômetro foram mais vantajosas que a técnica Tai Chi. O que contrasta com o estudo de Fuzhong et $\mathrm{al}^{22}$ que, ao comparar o Tai Chi com exercícios de fortalecimento e de alongamento, evidenciou maior eficácia do Tai Chi em relação à diminuição do risco de quedas e melhora das capacidades funcionais. Entretanto, nesse estudo, os autores esclarecem que o Tai Chi foi aplicado de maneira adaptada, levando em consideração as características da DP, isto é, foram escolhidos os seis movimentos mais simples do Tai Chi, realizados de forma repetitiva para aumentar o equilíbrio e melhorar a locomoção e, além disso, o treino da respiração foi inserido aos poucos durante os atendimentos. Ademais, esses autores observaram que o grupo de pacientes que fizeram Tai Chi obtiveram melhores resultados quanto à postura, a capacidade física, em relação aos participantes que fizeram treino individual de força e exercícios de alongamento.

O Tai Chi é um protocolo de 12 movimentos integrados com o objetivo de manter o equilíbrio e controle postural. Os participantes são estimulados a realizar movimentos simétricos, amplos e diagonais em pé, tais como, deslocamento do peso, deslocamento do centro de massa sobre a base de apoio, balanceios ântero-posteriores e laterais, associados com a respiração ${ }^{18}$. É uma atividade que pode ser realizada em grupo ou individual. Estudos ${ }^{18,22}$ que vem utilizando o Tai Chi no tratamento para indivíduos com DP vem apontando redução de discinesias, aumento da capacidade de adotar estratégias de balanceio (quadril e tornozelo) e de envolvimento com movimentos controlados, o que influencia diretamente no desempenho das funções de vida diária. Além disso, o Tai Chi, favoreceu o aumento da velocidade da marcha, associado ao aumento do comprimento do passo, que alivia os movimentos bradicinésicos.

Além desta atividade milenar, outra modalidade que vem se destacando como exercício físico em grupo para indivíduos com DP na melhora dos sinais motores, por exigir o equilíbrio e o ajuste postural é a Yoga. Ni, Mooney e Signorolea ${ }^{24}$ investigaram três programas de reabilitação que envolviam, treino de musculação, Yoga adaptada e educação em saúde para indivíduos com DP e encontraram benefícios não somente no treino de musculação, mas principalmente na Yoga, que favoreceu o aumento do equilíbrio, do tamanho da passada e da velocidade da marcha. A Yoga é caracterizada por posturas com transições rápidas e podem ser realizadas em posturas altas, baixas e intermediárias ${ }^{24}$. Essa atividade traz benefícios relacionados a força muscular, flexibilidade, equilíbrio, coordenação, melhorando a qualidade de vida do indivíduo. Pode ser adaptada de acordo com a demanda física de cada indivíduo. 
Apenas três estudos ${ }^{19,20,21}$ examinaram também os sintomas não motores de indivíduos com DP, tais como, o comprometimento cognitivo, a depressão e a apatia prejudicam as funções de atenção, planejamento motor, execução e a memória nos afazeres do dia a dia. O ponto em comum destes três estudos ${ }^{19,20,21}$ foi o uso da dança como instrumento na reabilitação das pessoas com DP. A dança é uma atividade atraente, motivacional, lúdica, e pode, portanto, ser usado para trabalhar a função tanto motora quanto não motora simultaneamente, além de ser uma atividade social $^{19,20,21}$.

No estudo de Hashimoto et a ${ }^{19}$ foi investigado os efeitos da dança na função motora e não motora por meio da comparação de uma atividade em grupo com dança, com outro grupo sem o uso da dança. A experiência desses autores mostrou que a dança foi efetiva na melhora da marcha e do equilíbrio, melhora do humor, aumento da motivação e diminuição da ansiedade. Volpe et $\mathrm{al}^{21}$ investigaram se a dança irlandesa é mais eficaz que exercícios de rotina em pessoas com DP e destacaram que a dança traz ritmo, movimentos amplos e rápidos e possibilidade de estar junto com outras pessoas, interferindo significativamente nos sinais não motores dos indivíduos. Já no estudo de Romenets et $\mathrm{al}^{20}$, comparou-se o tango com exercícios de rotina da fisioterapia, encontrando benefícios para $o$ congelamento durante a marcha, por trabalhar o equilíbrio, a mobilidade funcional, pois usa passos específicos ritmicamente para frente e para trás, além de estimular a memória e controle da atenção.

Embora a dança seja um modo popular de atividade física e sua prática pode estar associada com melhorias na mobilidade, equilíbrio e qualidade de vida, não está estabelecido na literatura se a dança é mais eficaz a longo prazo, em comparação com exercícios terapêuticos convencionais ${ }^{21,22,23}$. Além disso, não se sabe ainda qual o tipo de música, gênero de dança e dosagem dessa atividade é mais adequada para atingir os benefícios terapêuticos em indivíduos com DP, porém, sabe-se que a dança favorece atividades de dupla tarefa que contribui na ativação das regiões que normalmente exibem baixa atividade na $\mathrm{DP}^{20}$.

Por fim, existem várias abordagens terapêuticas para indivíduos com DP e consensualmente reforçam a importância da atividade física, do exercício físico em $\mathrm{si}^{28}$. No estudo de Carvalho et $\mathrm{al}^{23}$ foi realizado uma comparação entre programas de reabilitação em grupo que envolviam atividades de flexibilidade, resistência, circuitos aeróbicos, uso de pistas sensoriais e exercícios calistênicos, e foi constatado que todas as abordagens contribuíram na melhoria da saúde física e mental dos pacientes com DP. Vale destacar que, é preciso que haja o controle regular dos progressos e que os indivíduos sejam cuidadosamente ajustados às necessidades para o sucesso terapêutico ${ }^{29}$.

Embora a presente revisão aponte estratégias de intervenção que trazem benefícios terapêuticos a indivíduos com DP, traz limitações em não encontrar afirmação sobre qual a melhor modalidade de exercício, pois os estudos apresentados abordam grupos com diferentes intervenções levando a benefícios diferenciados. Mais estudos com boa qualidade metodológica ajudam a triar e eleger a modalidade que se destaca entre os benefícios motores e não motores de indivíduos com DP.

\section{Conclusão}

O exercício físico parece trazer efeitos positivos para indivíduos com DP. As abordagens terapêuticas têm procurado solucionar tanto os sinais motores quanto os não motores da DP. Enquanto as atividades que envolvem movimentos amplos, repetitivos, com resistência e circuitos aeróbicos enfatizam os sintomas motores, a dança tem sido utilizada para englobar os sintomas não motores de indivíduos acometidos com a DP. Contudo, a estratégia de realizá-las em grupo é o diferencial por associar a vivência de indivíduos que apresentam a mesma enfermidade, potencializando seus benefícios.

\section{Declaração de conflitos de interesses}

Os autores do artigo afirmam que não houve nenhuma situação de conflito de interesse, tais como propostas de financiamento, emissão de pareceres, promoções ou participação em comitês consultivos ou diretivos, entre outras, que pudessem influenciar no desenvolvimento do trabalho.

\section{Referências}

1- ALLEN, N. E. et al. The effects of an exercise program on fall risk factors in people with Parkinson's disease: a randomized controlled trial. Moviment Disorders, v. 25, n. 9, p. 1217-1225, 2010.

2- LANA, R. C.; POLESE, J. C. Condicionamento cardiorrespiratório na doença de Parkinson. In: Associação Brasileira de Fisioterapia Neurofuncional; Garcia CSNB, Fachinetti LD, organizadoras. PROFISIO Programa de Atualização em Fisioterapia Neurofuncional: Ciclo 4. Porto Alegre: Artmed Panamericana; 2016. p. 53-83. (Sistema de Educação Continuada a Distância, v. 1).

3- PAGNUSSAT, A. S.; KLEINER, A. F.; MARCHESE, R. R.; GALLI, M. Reabilitação da marcha do paciente com doença de Parkinson. In: Associação Brasileira de Fisioterapia Neurofuncional; Garcia CSNB, Facchinetti LD, organizadoras. PROFISIO Programa de Atualização em Fisioterapia Neurofuncional: Ciclo 4. Porto Alegre: Artmed Panamericana; 2016. p. 9-52. (Sistema de Educação Continuada a Distância, v.1).

4- GOULART, F. R.P. et al. O impacto de um programa de atividade física na qualidade de vida de pacientes com doença de Parkinson. Revista Brasileira de Fisioterapia, v. 9, n. 1, p. 49-55, 2005. 
5- KEUS, S. H. J. et al. Evidence-Based Analysis of Physical Therapy in Parkinson's Disease with Recommendations for Practice and Researchers. Moviment Disorders, v. 22, n. 4, p. 451-446, 2007.

6- CRUISE, K. E. et al. Exercise and Parkinsons: benefits for cognition and quality of life. Acta Neurology Scandian, v. 123, p. 13-19, 2011.

7- SHULMAN, L. M. et al. Randomized Clinical Trial of 3 Types of Physical Exercise for Patients With Parkinson Disorder Journal Neurology, v. 70, n. 2, p. 183-190, 2013.

8- AMANO, S. et al. The effect of Tai Chi exercise on gait initiation and gait performance in persons with Parkinson's disease. Parkinson Res Disorder, v. 19, p. 955-960, 2013.

9- BRAUN, S. et al. Rehabilitation with mental practice has similar effects on mobility as rehabilitation with relaxation in people with Parkinson's disease: a multicentre randomised trial. Journal Physical, v. 57, p. 27-34, 2011.

10- RODRIGUES DE PAULA, F. et al. Exercício aeróbio e fortalecimento muscular melhoram o desempenho funcional na doença de Parkinson. Fisioterapia e Movimento, v. 24, p. 379-388, 2011.

11- MORRIS, M. E. et al. Falls and mobility in Parkinson's disease: protocol for a randomised controlled clinical trial. BMC Neurol, v. 11, n. 3, p. 2-8, 2011.

12- MENDES, K. D. S.; SILVEIRA, R. S. C. P.; GALVAO, C. M. Revisão integrativa: método de pesquisa para a incorporação de evidências na saúde e na enfermagem. Texto contexto - enfermagem, Florianópolis, v. 17, n. 4, Dec. 2008. Disponível em 〈http://dx.doi.org/10.1590/S0104-07072008000400018〉. Acesso em: 25 nov. 2017.

13- SAMPAIO, R. F. et al. Estudos de revisão sistemática: um guia para síntese criteriosa científica. Revista Brasileira de Fisioterapia, v. 11, n. 1, p. 83-89, 2007.

14- GREENHALGH, T. How to read a paper: papers that summarise other papers (systematic reviews and metaanalyses). BMJ, v. 315, p. 672-675, 1997.

15- OXMAN, A. D; SACKETT, D. L.; GUYATT, G. H. Users' guide to the medical literature I: how to get started. JAMA, v. 270, p. 2093-2095, 1993.

16- OXMAN, A. D. COOK, D. J.; GUYATT, G. H. Evidence-based medicine working group. User's guide to the medical literature: IV. How to use an overview. JAMA, v. 272, p. 1367-1371, 1994.

17. MAHER, C. G.; SHERRINGTON, C.; HERBERT, R D.; MOSELEY, A M.; ELKINS, M. Reliability of the PEDro scale for rating quality of randomized controlled trials. Physical Therapy, v. 83, p. 713-721, 2003.
18- ZHANG, Y. et al. Effects of Tai Chi and Multimodal Exercise Training on Movement and Balance Function in Mild to Moderate Idiopathic Parkinson Disease. American Journal Physical Medicine Rehabilitation, v. 94, n. 10, p. 922-929, 2015.

19- HASHIMOTO, H. et al. Effects of dance on motor functions,cognitive functions, and mental symptomsof Parkinson's disease: A quasi-randomizedpilot trial. Complement Therapy Medicine, v. 23, p. 210-219, 2015.

20- ROMENETS, S. R. et al. Tango for treatment of motor andnon-motor manifestations in Parkinson'sdisease: A randomized control study. Complement Therapy Medicine, v. 23, p. 175-184, 2015.

21- VOLPE, D. et al. A comparison of Irish set dancing and exercises for people with Parkinson's disease: A phase II feasibility study. BMC Geriatrics, v. 13, n. 54, p. 2-6, 2013.

22- FUZHONG, L. et al. Tai Chi and Postural Stability in Patients with Parkinson's Disease. The New England Journal Medicine, v. 6, n. 9, p. 511-519, 2012.

23- CARVALHO, A. et al. Comparison of strength training, aerobic training, and additional physical therapy as supplementary treatments for Parkinson's disease: pilot study. Clinical Intervew Aging, v. 10, p. 183-191, 2015.

24- NI M.; MOONE K.; SIGNORIELA J.F. Controlled pilot study of the effects of power yoga in Parkinson's disease. Complement Therapy Medicine, v. 25, p. 126-131, 2016.

25- HENDERSON, L.; KENNARD, L.; CRAWFORD, T. J.; et al. Scales for rating motor impairment in Parkinson's disease: studies of reliability an convergent validity. Journal Neurology Neurosurgery Psychiatry, v. 54, p. 18-24, 1991.

26- HORTA, W. Escalas clínicas para avaliação de pacientes com doença de Parkinson. In: Meneses MS, Teive HAG. Doença de Parkinson: aspectos clínicos e cirúrgicos. Rio de Janeiro: Guanabara Koogan; 1996. cap.8, p.83-96.

27- JENKINSON, C.; PETO, V.; FITZPATRICK, R. et al. Self-reported functioning and well-being in patients with Parkinson's disease: comparison of the short-form health survey (SF-36) and the Parkinson's Disease Questionnaire (PDQ-39). Age Ageing, v24, p. 505-509, 1995.

28- NIMWEGEN, M. et al. Promotion of physical activity and fitness in sedentary patients with Parkinson's disease: randomised controlled trial. BMJ, v. 576, p. 1-11, 2013.

29- HEUVEL, M. R. C. V. et al. The effects of augmented visual feedback during balance training in Parkinson's disease: study design of a randomized clinical trial. BMC Neurology, v. 13, n. 137, p. 2-8, 2013. 
30- ALBUQUERQUE, C. P. et al. Grupo de atividades de vida diária: influência do procedimento em pacientes adultos com acidente vascular encefálico isquêmico. Acta Fisiatrica, v. 18, n. 2, p. 71-74. 